\title{
Psychological wellbeing and incident frailty in men and women: The English Longitudinal Study of Ageing
}

\author{
Catharine R Gale ${ }^{1,2}$, Cyrus Cooper ${ }^{1}$, lan J Deary ${ }^{2}$, and Avan Aihie Sayer ${ }^{1}$ \\ ${ }^{1} \mathrm{MRC}$ Lifecourse Epidemiology Unit, University of Southampton, Southampton, UK \\ ${ }^{2}$ Centre for Cognitive Ageing and Cognitive Epidemiology, Dept of Psychology, University of \\ Edinburgh, Edinburgh, UK
}

\section{Abstract}

\begin{abstract}
Background-Observations that older people who enjoy life more tend to live longer suggest that psychological wellbeing may be a potential resource for healthier ageing. We investigated whether psychological wellbeing was associated with incidence of physical frailty.
\end{abstract}

\begin{abstract}
Methods-We used multinomial logistic regression to examine the prospective relation between psychological wellbeing, assessed using the CASP-19 questionnaire that assesses perceptions of control, autonomy, self-realization and pleasure, and incidence of physical frailty or pre-frailty, defined according to the Fried criteria, in 2557 men and women aged 60 to over 90 years from the English Longitudinal Study of Ageing.
\end{abstract}

\begin{abstract}
Results-Men and women with higher levels of psychological wellbeing were less likely to become frail over the 4-year follow-up period. For a standard deviation higher score in psychological wellbeing at baseline, the relative risk ratio (95\% confidence interval) for incident frailty, adjusted for age, sex and baseline frailty status, was $0.46(0.40,0.54)$. There was a significant association between psychological wellbeing and risk of pre-frailty: $0.69(0.63,0.77)$. Examination of scores for hedonic (pleasure) and eudaimonic (control, autonomy and selfrealization) wellbeing showed that higher scores on both were associated with decreased risk. Associations were partially attenuated by further adjustment for other potential confounding factors but persisted. Incidence of pre-frailty or frailty was associated with a decline in wellbeing, suggesting that the relationship is bi-directional.
\end{abstract}

Conclusions-Maintaining a stronger sense of psychological wellbeing in later life may protect against the development of physical frailty. Future research needs to establish the mechanisms underlying these findings.

\section{INTRODUCTION}

Maintaining a strong sense of psychological wellbeing in the face of the changes and losses of later life is generally considered a crucial part of "healthy" ageing (Baltes and Baltes, 1990; Rowe and Kahn, 1998). In recent years there has been growing evidence to suggest that wellbeing may also be a potential resource for generally ageing well. Prospective studies have found that older people with greater wellbeing — whether assessed according to the hedonic view of wellbeing as positive affect or satisfaction with life, or according to the eudaimonic view of wellbeing as sense of purpose, autonomy or meaning in life - are less likely to develop problems with mobility or other activities of daily life (Boyle et al.

Correspondence to: Dr Catharine Gale, MRC Lifecourse Epidemiology Unit, Southampton General Hospital, Southampton, SO16 6YD, UK. Tel: 44 (0)23 80764080. Fax: 44 (0)23 704021. crg@mrc.soton.ac.uk.

Declaration of interest None. 
2010; Collins et al. 2008; Ostir et al. 2000), show a slower decline in perceptual speed (Gerstorf et al. 2007), and make a better recovery in terms of motor, cognitive and functional status after a stroke (Ostir et al. 2008). Older people with a more positive attitude to their own ageing report better functional health at subsequent follow-ups (Levy et al. 2002). In a meta-analysis of 35 prospective studies where greater positive wellbeing was linked with a reduced risk of mortality both in healthy populations and in those already ill at baseline, the survival benefit associated with greater wellbeing was particularly marked in people aged 60 and over (Chida and Steptoe, 2008). In all of these studies, with the exception of that by Levy et al where there was no control for the potentially confounding effect of depressive symptoms, the protective effect of positive wellbeing persisted after adjustment for negative affect, and so was not due merely to the absence of symptoms of depression.

Frailty is a clinically recognizable syndrome in older people whose core feature is an increased vulnerability to stressors due to impairments in multiple, inter-related systems, decreased physiological reserves and a decline in the ability to maintain homeostasis (Bergman et al. 2007; Fried et al. 2001). It is common (Syddall et al. 2010) and associated with numerous adverse outcomes, including falls, disability, hospitalization, institutionalization and death. There is currently no universally accepted model or definition of frailty (Rockwood and Bergman, 2012; Rodriguez-Manas et al. 2013), but it is generally agreed that its causes are complex and likely to involve both biological and psychosocial mechanisms (Rockwood et al. 1994; Walston et al. 2006). Evidence as to whether psychological wellbeing might be a protective factor as regards risk of frailty in later life is sparse, but in two prospective studies, older people who reported high levels of positive affect had a reduced likelihood of becoming physically frail (Ostir et al. 2004; Park-Lee et al. 2009). Whether other dimensions of psychological wellbeing are similarly protective as regards risk of frailty is unknown. In a cross-sectional analysis of data from the Canadian Study of Health and Aging, higher scores on an index of frailty--comprising 33 health deficits-was associated with lower scores on the Ryff Psychological Wellbeing scale (Andrew et al. 2012). Of the six subscales that make up this measure-named autonomy, personal growth, environmental mastery, positive relations, purpose in life and selfacceptance--only purpose in life and autonomy were not associated with frailty score. Whether these findings reflect the effect of these dimensions of wellbeing on frailty risk or the impact of increasing frailty on wellbeing is unclear.

The English Longitudinal Study of Ageing (ELSA) is a large population-based sample of older men and women. We used these data to investigate the 4-year prospective association between scores on the CASP-19, a measure of psychological wellbeing that assesses perceptions of control, autonomy, self-realization and pleasure (Hyde et al. 2003), and risk of incident physical frailty in men and women aged 60 to over 90 years.

\section{METHODS}

\section{Participants}

The data for this study come from the English Longitudinal Study of Ageing (ELSA). The sample for ELSA was based on people aged $\geq 50$ years who had participated in the Health Survey for England in 1998, 1999 or 2001 (Marmot et al. 2011) (Steptoe et al. 2012a). It was drawn by postcode sector, stratified by health authority and proportion of households in non-manual socioeconomic groups. 11,392 people participated in Wave 1 in 2002-3. At Wave 2 in 2004-5 and at Wave 4 in 2008-9 participants who completed the main interview were invited to have a visit from a nurse that included measurements of physical function, anthropometry and blood sampling. Nurse visits are conducted at alternate follow-up surveys so data on these measurements were not collected at Wave 3. Ethical approval was 
obtained from the Multicentre Research and Ethics Committee. Participants gave written informed consent.

\section{Measures}

Maximum handgrip strength was measured three times on each side using a dynamometer; the best of these measurements was used for analysis. Height and weight were measured with a portable stadiometer and electronic scales respectively. Body mass index (BMI) was calculated as weight (in kilograms)/height (in metres). ${ }^{2}$ Gait speed was assessed in participants aged 60 and over by measuring the time taken to walk a distance of 8 feet at usual pace; the timed walk was repeated and the mean of the two measurements was calculated. Participants responded to three questions about the frequency with which they did vigorous exercise (eg running, swimming, cycling, tennis, aerobics or gym workout, digging), moderate exercise (eg gardening, cleaning the car, walking at a moderate pace, dancing, floor or stretching exercise) and mild exercise (eg vacuuming, laundry, home repairs). Each question had four response options (more than once a week, once a week, one to three times a month, hardly ever or never). These questions about physical activity were extracted from a validated physical activity interview used in the Health Survey for England (Joint Health Surveys Unit, 2007) We ranked the combinations of responses to these questions according to the amount and intensity of exercise involved to provide a summary estimate of usual physical activity, whereby higher scores indicated more frequent, vigorous activity. Symptoms of depression were assessed using the eight-item version of the Center for Epidemiologic Studies Depression Scale (CES-D) (Steffick and The HRS working group, 2000). We used these data, together with information on participants' weight at the initial survey, to derive indicators of pre-frailty and frailty at Wave 2 and at Wave 4 in men and women aged $\nsucceq 60$ years using the Fried criteria; there are unintentional weight loss, weakness, self-reported exhaustion, slow walking speed and low physical activity (Fried $e t$ al. 2001). Pre-frailty was defined as the presence of one or two of these criteria. Frailty was defined as the presence of three or more of these criteria. These criteria were originally validated in the Cardiovascular Health Study and the Women's Health and Aging Studies (Bandeen-Roche et al. 2006; Fried et al. 2001) and have subsequently been validated in other cohorts (Avila-Funes et al. 2008; Bouillon et al. 2012). We operationalized these criteria using definitions which were identical or very similar to those used in the original phenotype of frailty studies (Bandeen-Roche et al. 2006; Fried et al. 2001): weight loss was defined as either loss of $\geq 10 \%$ of body weight since the initial survey (for frailty at Wave 2) or since Wave 2 (for frailty at Wave 4), or current BMI $<18.5 \mathrm{~kg} / \mathrm{m}^{2}$; weakness was defined as maximum grip strength in the lowest $20 \%$ of the distribution, after taking sex and BMI into account; exhaustion was considered to be present if the participant gave a positive response to either of the CES-D questions 'Felt that everything I did was an effort in the last week' or 'Could not get going in the last week'; slow walking speed was defined as a walking speed in the lowest $20 \%$ of the distribution, after taking account of sex and height; as in some previous studies of frailty (Espinoza et al. 2012; Ostir et al. 2004) participants who did not perform the timed walk because they were unable to walk alone or had health restrictions were also categorised as having slow walking speed; low physical activity was defined as physical activity in the lowest sex-specific $20 \%$ of the distribution. In the case of weakness, exhaustion, or physical activity the definitions and cut-points used were identical to those used in the original phenotype of frailty studies (Bandeen-Roche et al. 2006; Fried et al. 2001):

Participants were asked whether a doctor had ever told them that they had any of the following conditions: high blood pressure/hypertension, angina, heart attack, congestive heart failure, diabetes or high blood sugar, a stroke, chronic lung disease, asthma, arthritis or rheumatism, osteoporosis, or cancer. We used this information to calculate the number of 
chronic physical diseases present. Participants took tests of cognitive function (Steel et al. 2003) as follows: immediate and delayed verbal memory (recall of 10 aurally-presented nouns) was assessed using word lists developed for the US Health and Retirement Study; prospective memory (remembering to do a specific task) and attention (letter cancellation task) were assessed using tests previously used in the MRC Cognitive Function and Ageing Study (Brayne et al. 1998); and executive function was assessed using a test of verbal (semantic) fluency (naming as many animals as possible in 60 seconds) taken from the CAMCOG-R (Roth et al. 1999). A total cognitive function score was calculated by summing scores on these tests. Socioeconomic status was indexed by total household wealth, including savings and investments, value of any property or business assets, net of debt, excluding pension assets. Household wealth has been identified as the most accurate indicator of long-term socioeconomic circumstances in ELSA (Banks et al. 2003). Participants provided information about current smoking status and were categorized into three groups (never smoked, ex-smokers and current smokers).

Psychological wellbeing was assessed using the CASP-19 quality of life questionnaire that consists of 19 items covering four theoretical domains, control, autonomy, self-realization and pleasure (Hyde et al. 2003). Examples include: "I feel free to plan for the future" (control), "I feel that I can please myself what I do" (autonomy), "I choose to do things that I have never done before" (self-realization) and "I enjoy the things that I do" (pleasure). The response to each item is a four-point Likert scale coded 0 to 3 . Total scores range from 0 to 57 with higher scores indicating greater psychological wellbeing. In order to assess whether hedonic and eudaimonic dimensions of psychological wellbeing had similar associations with risk of frailty we used scores on the 4-item pleasure subscale as a measure of hedonic wellbeing and combined total score on the remaining 15 items as a measure of eudaimonic wellbeing, as has been done previously (Steptoe et al. 2012b). Cronbach alpha values in the study sample were 0.88 for the total score, 0.68 for hedonic wellbeing and 0.78 for eudaimonic wellbeing, indicating good internal consistency.

During the visit by the nurse, blood samples were taken from participants except those who were not willing to give written consent, those with clotting or bleeding disorders and those taking anti-coagulant drugs. Fasting samples (defined as no food or drink except water for the past 5 hours) were taken where possible (67\% of participants). Samples were assayed for C-reactive protein and fibrinogen at the Royal Victoria Infirmary, Newcastle-upon-Tyne, UK. Detailed information on the technicalities of the blood analysis, the internal quality control, and the external quality assessment for the laboratory that carried it out are given in the 2004 Health Survey for England technical report as both the Health Survey for England and HSE and ELSA used the same laboratory, and the same guidelines and protocols for blood analysis (Graig et al. 2006).

\section{Analytical sample}

Of the 5918 study members aged $\searrow 60$ years who were interviewed at Wave 2 in 2004-5, 5377 agreed to be visited by a nurse $(91 \%)$. Of these, 3454 were interviewed and visited by a nurse at Wave 4 in 2008-9 after a mean follow-up period of 3.93 years. The present analysis is based on 2557 people who had complete data on psychological wellbeing and baseline covariates at Wave 2 and frailty at Wave 4 . To investigate possible bias due to missing data, we used multiple multivariate imputation to impute values in any covariates with missing values (Royston, 2004). Imputation models included psychological wellbeing, components of frailty and the potential confounding variables. We used switching regression in STATA software (Royston, 2004) and carried out 10 cycles of regression switching and generated 10 imputation data sets. The multiple multivariate imputation approach creates a number of copies of the data (in this case 10 copies) each of which has missing values 
imputed based on available data and with an appropriate level of randomness using chained equations. The final estimates are obtained by averaging across the estimates from each of these 10 data sets using Rubin's rules and taking into account the uncertainty in the imputation as well as uncertainty due to random variation. Effect sizes obtained after imputation of missing information were essentially the same as those obtained from the sample with complete data, so we present results based on the sample with complete data.

\section{Statistical analysis}

We used ANOVA and Chi-square test to examine differences in baseline characteristics according to the presence of pre-frailty or frailty at follow up. We used multinomial logistic regression to examine the relation between total score for psychological wellbeing at baseline and risk of incident pre-frailty or frailty, controlling for potentially confounding factors (age, sex, household wealth, smoking status, number of chronic physical diseases, BMI, depressive symptom score, cognitive function, and frailty status at baseline (i.e. whether participants had no, one or two, or three or more components of frailty)). In order to assess whether hedonic or eudaimonic dimensions of psychological wellbeing differed in their relation with frailty risk, we repeated these analyses using scores for hedonic and eudaimonic wellbeing in place of total score. In a subset of participants with data on blood concentrations of the inflammatory markers $C$-reactive protein and fibrinogen $(n=1786)$ we examined the effect on the associations of further adjustment for these factors as there is some evidence to link both of them with lower levels of wellbeing(Steptoe et al. 2012b)and with risk of frailty (Barzilay et al. 2007; Gale et al. 2013; Puts et al. 2005). We used linear regression to examine whether the onset of pre-frailty or frailty in those who were initially not frail was associated with a change in psychological wellbeing at follow-up. Analyses were weighted using probability weights supplied with the data to correct for any systematic differences in response rates across subgroups and to take account of the complex design of the survey (Steptoe et al. 2012a). Detailed descriptions of these weights and their calculation can be found in the technical reports on the study available at www.ifs.org.uk/elsa.

\section{Results}

Table 1 shows the baseline characteristics of the 2557 men and women in the study according to whether they were not frail, pre-frail or frail at follow-up. The weighted percentage of participants who were pre-frail or frail at follow-up was $43 \%$ and $14 \%$ respectively. Degree of frailty at follow-up, as indicated by whether participants were classified as not frail, pre-frail or frail at that time, was associated with older age, greater likelihood of being in the lowest quintile for household wealth, having more chronic physical diseases, poorer cognitive function, greater prevalence of depression, having a higher BMI, poorer psychological wellbeing and having more indicators of frailty at baseline. There was no difference in sex distribution between those who were not frail and those who were pre-frail at follow-up, but there were a significantly higher proportion of women among those who were frail. Compared to those who were not frail at follow-up, those who were pre-frail were slightly more likely to have smoked at baseline, but there were no differences in baseline smoking status between those who were pre-frail and those who were frail at follow-up.

Preliminary analyses showed that the relationship between psychological wellbeing and risk of incident pre-frailty or frailty did not differ between the sexes or by age ( $\mathrm{p}$ for interaction terms >0.5). Multivariate multinomial logistic regression analyses of frailty risk were therefore carried out in men and women together, adjusting for sex and age.

Table 2 show relative risks for incident pre-frailty and frailty in men and women for a standard deviation higher score in psychological wellbeing (both total score and scores on 
the two subscales, hedonic and eudaimonic wellbeing). Results are shown adjusted first for age, sex and baseline frailty status, and then with further adjustment for the other potential confounding variables, household wealth, depressive symptoms, cognitive function, body mass index, smoking, and number of chronic physical diseases. In models adjusting for age, sex and baseline frailty status there were significant inverse associations between total score for psychological wellbeing at baseline and risk of incident frailty: for a standard deviation increase in psychological wellbeing the relative risk ratio (RR) (95\% confidence interval) for incident frailty was $0.46(0.40,0.54)$. Further adjustment for other potential confounding factors partially attenuated the association: RR $(95 \% \mathrm{CI}) 0.62(0.52,0.77)$. There was a significant association between total score for psychological wellbeing and risk of incident pre-frailty: for a standard deviation increase in psychological wellbeing the relative risk ratio (95\% confidence interval) for incident pre-frailty was $0.69(0.63,0.77)$. Further adjustment for other potential confounding factors partially attenuated this association: RR (95\% CI) $0.79(0.71,0.89)$. We repeated these analyses to compare whether risk of frailty varied depending on whether hedonic or eudaimonic dimensions of wellbeing were the predictor. Higher scores on both dimensions were associated with decreased risk of frailty or prefrailty.

In the analyses described above, in order to maximise our sample size we included participants who were pre-frail or frail and adjusted for frailty status at baseline. To check for the possibility of reverse causation whereby psychological wellbeing at baseline might have been adversely affected in those who already had one or more components of frailty, we repeated our analyses after excluding all such individuals. In the remaining sample of 1409 men and women, none of whom were pre-frail or frail at baseline, associations between psychological wellbeing and risk of incident frailty were very similar to those found in the full sample: for a standard deviation increase in psychological wellbeing at baseline the multivariate-adjusted risks of incident pre-frailty or frailty were $0.77(0.66$, $0.89)$ and $0.58(0.41,0.84)$ respectively.

In all models, increased age, female sex, lower household wealth, higher BMI, greater number of chronic physical diseases, and poorer cognition were independent risk factors for incident frailty and pre-frailty (data not shown).

In a subset of participants who had data on blood concentrations of the inflammatory markers C-reactive protein and fibrinogen at baseline $(n=1786)$, we repeated the multivariate analyses with additional adjustment for these variables. This adjustment had no effect on the associations between baseline psychological wellbeing scores and risk of pre-frailty or frailty at follow-up (data not shown) either in the whole sample or when men and women were analysed separately.

To illustrate how risk of pre-frailty or frailty at follow-up varied according to level of psychological wellbeing at baseline, figure 1 shows fully-adjusted relative risk ratios according to quarters of the distribution of psychological wellbeing scores at baseline, using those in the lowest quarter of the distribution as the reference group (Figure 1).

At the time of the baseline survey, levels of psychological wellbeing were significantly lower in those with more components of frailty ( $\mathrm{p}$ for linear trend $<0.0001$ ). To investigate whether this cross-sectional association might be bi-directional in effect, we used the sample of 1409 people who were neither pre-frail nor frail at baseline to examine whether the onset of pre-frailty or frailty during the follow-up period was associated with a change in psychological wellbeing since baseline. In regression analyses with adjustment for age, sex, and psychological wellbeing at baseline, scores for psychological wellbeing at follow-up were 1.58 points $(95 \%$ CI $0.93,2.22)$ lower in those who had become pre-frail and 3.70 
points $(1.99,5.41)$ lower in those who had become frail compared to those who were not frail. These associations were only slightly attenuated when further adjusted for the potential confounding factors, depressive symptom score, cognitive function, household wealth, number of chronic illnesses, BMI and smoking status at baseline. After these adjustments scores for psychological wellbeing at follow-up were 1.30 points $(95 \%$ CI $0.65,1.96)$ lower in those who had become pre-frail and 2.30 points $(1.22,4.77)$ lower in those who had become frail compared to those who were not frail. Separate analysis of the eudaimonic and hedonic dimensions of psychological wellbeing showed that those who had become pre-frail or frail showed a relative decline on both dimensions on wellbeing since the baseline survey (data not shown).

\section{Discussion}

In this large longitudinal study of people aged 60 to over 90 years, higher scores on a measure of psychological wellbeing that assesses control, autonomy, self-realisation and pleasure were associated with an increased risk of incident pre-frailty and frailty in both men and women. Adjustment for a range of potential confounding factors, including depressive symptoms, household wealth, cognitive function, number of chronic physical illnesses, BMI, smoking, inflammatory markers, age and sex, and baseline frailty status had only small attenuating effects on these associations, and estimates changed little when analyses were repeated excluding all those who were pre-frail or frail at baseline. Separate examination of scores for hedonic (pleasure) and eudaimonic (control, autonomy and self-realization) dimensions of the psychological wellbeing measure showed that higher scores on both were associated with decreased risk of frailty or pre-frailty. These results suggest that psychological wellbeing may be a protective factor. We also found an effect in the reverse direction in that the incidence of either pre-frailty or frailty in those initially not frail was associated with a relative decline in psychological wellbeing since the baseline survey.

Only two previous studies have examined the prospective association between psychological wellbeing in older people and risk of becoming frail. In a cohort of 1500 Mexican Americans, high levels of positive affect were associated with a lower risk of incident frailty (Ostir et al. 2004). One limitation of this study is that no adjustment was made for depressive symptoms so it is impossible to be certain whether the findings reflect a potentially protective effect of positive affect or merely the absence of negative affect. In another US cohort based solely on women - the Caregiver-Study of Osteoporotic Fractures - high levels of positive affect were predictive of a reduced risk of incident frailty independently of depressive symptoms (Park-Lee et al. 2009). The results of the present study shows that it may be not only the hedonic aspects of wellbeing such as the experience of happiness and pleasure that protects against the development of frailty, but also eudaimonic aspects such as a sense of autonomy, control, purpose and fulfilment. No previous study has examined whether psychological wellbeing is predictive of the state of pre-frailty. Consistent with the hypothesis that pre-frailty and frailty represent degrees of severity in the same chronic process, we found that psychological wellbeing, along with other risk factors examined, was predictive of both.

It is important to consider whether our results showing an apparently protective effect of psychological wellbeing could have arisen due to reverse causation. In accordance with Fried (Bandeen-Roche et al. 2006; Fried et al. 2001) physical frailty was defined as the presence of 3 or more of criteria (unintentional weight loss, weakness, self-reported exhaustion, slow walking speed and low physical activity) and pre-frailty was defined as the presence of 1 or 2 of these criteria. In looking at risk of developing frailty or pre-frailty at follow-up, we adjusted for the number of these criteria that were present at baseline, and for symptoms of depression and BMI at baseline together with other potential confounding 
factors. Associations between wellbeing and later frailty or pre-frailty were only partially attenuated by these adjustments, and persisted when people who were pre-frail or frail at baseline were excluded, but might factors such as weakness, slow walking speed and low physical activity have had an adverse impact on psychological wellbeing prior to baseline, before they reached a level where they counted as frailty criteria? Evidence on this is sparse. In a meta-analysis of longitudinal data from five cohorts in the HALCyon (Healthy Ageing across the Lifecourse) programme, better performance on measures of grip strength, walking speed, timed get up and go speed and chair rise speed were all associated with slightly higher scores on the Warwick Edinburgh mental wellbeing scale - a measure of both hedonic and eudaimonic wellbeing -- a few years later, but the direct of effect in these associations could not be accurately determined (Gale et al. in press). So far as we are aware, there is no previous longitudinal evidence as to whether poorer physical capability per se leads to a decline in wellbeing in older people, though the results of the current study suggest that the onset of pre-frailty or frailty-assessed in part by the presence of weakness or slow walking speed-is predictive of lower psychological wellbeing at follow-up than would be expected from initial levels. This difference persisted after adjustment for depressive symptoms, cognitive function and other potential confounding factors. It has been suggested that the transition from robust health to increasing frailty can pose psychological challenges as individuals have to adjust to the losses and changes inherent in such a transition (Fillit et al. 2009). This may account for the apparent decline in psychological wellbeing found here. Further follow-up is needed for accurate determination of the trajectory of wellbeing in these frail older people.

The mechanisms whereby psychological wellbeing might influence risk of frailty, or indeed other aspects of health, are not fully understood (Steptoe et al. 2009). Health behaviours such as exercise, diet and smoking may provide one potential explanation as they can be influenced by emotional state (Kiecolt-Glaser et al. 2002). Previous analyses using the ELSA cohort have shown that higher levels of psychological wellbeing are associated with being more physically active and with a lower likelihood of smoking. In the present paper it seemed inappropriate to adjust for physical activity level given that low physical activity is one of the frailty criteria, but when we did so (data not shown) it had very little attenuating effect on the associations. We included adjustment for smoking but this behaviour too did not account for the link between wellbeing and risk of frailty. No data were available on diet at baseline. Another possibility is that direct psychobiological mechanisms underlie the association. Higher levels of psychological wellbeing have been associated in crosssectional surveys with lower concentrations of inflammatory markers and cortisol.(Steptoe et al. 2009) There is some evidence from prospective studies that higher levels of inflammatory markers or a higher ratio of cortisol to dehydroepiandrosterone sulphate may be risk factors for frailty (Barzilay et al. 2007; Baylis et al. 2012; Puts et al. 2005; Reiner et al. 2009). A previous paper using ELSA data reported cross-sectional associations between higher levels of psychological wellbeing and lower blood levels of the inflammatory markers, C-reactive protein and fibrinogen, and-in men only--higher blood levels of dehydroepiandrosterone sulphate at Wave 4 (Steptoe et al. 2012b). We had no information on concentrations of cortisol or dehydroepiandrosterone sulphate in our participants at the Wave 2 baseline, but adjustment for levels of the inflammatory markers, C-reactive protein and fibrinogen had no effect on our estimates for the relations between psychological wellbeing and either frailty or pre-frailty.

The strengths of our study include the large sample size, the fact that it is representative of the community-dwelling English population aged 60 and over (Taylor et al. 2003), our use of a prospective study design in which we assessed incident cases of pre-frailty or frailty that occurred after the measurement of psychological wellbeing, and the fact that we had information on a range of potential confounding factors. There are also some weaknesses. 
Firstly, we were not able to assess whether psychological wellbeing assessed prior to the development of pre-frailty or frailty might promote the transition to a less frail state. Whereas transitions to a state of greater frailty are more common than movement in the reverse direction, there is sufficient evidence of the latter to suggest that frailty is potentially reversible, at least in the less severe stage (Espinoza et al. 2012; Gill et al. 2006). Secondly, not all the participants in the baseline survey gave a blood sample (77\% of those interviewed); non-responders to the blood sample tended to be older and in poorer health. However, in view of the fact that adjustment for concentrations of inflammatory factors in the subsample with these data had no attenuating effect on the psychological wellbeingfrailty associations, we think it unlikely that these findings would be very different if we had data on inflammatory factors for the entire study sample. Furthermore, all analyses have been weighted to reduce any potential bias arising from differential non-response.

In this prospective study of men and women aged 60 to over 90 years, those who scored higher on a measure of psychological wellbeing that assessed perceptions of control, autonomy, self-realisation and pleasure had a lower risk of becoming physical frail during follow-up. Future research needs to investigate the mechanisms underlying this finding.

\section{Acknowledgments}

We are grateful to the UK Data Archive for supplying the ELSA data. The original data creators, depositors or copyright holders, the funders of the data collections and the UK Data Archive bear no responsibility for the analysis or interpretation presented here.

The Centre for Cognitive Ageing and Cognitive Epidemiology is funded by the Biotechnology Sciences Research Council, the Engineering and Physical Sciences Research Council, the Economic and Social Research Council, the Medical Research Council and the University of Edinburgh as part of the cross-council Lifelong Health and Wellbeing initiative (G0700704/84698).

Funding: Medical Research Council - MC-A620-5TF00; Lifelong Health and Wellbeing Initiative G0700704/84698).

\section{References}

Andrew MK, Fisk JD, Rockwood K. Psychological well-being in relation to frailty: a frailty identity crisis? International Psychogeriatrics. 2012; 24:1347-1353. [PubMed: 22436131]

Avila-Funes JA, Helmer C, Amieva H, Barberger-Gateau P, Le GM, Ritchie K, Portet F, Carriere I, Tavernier B, Gutierrez-Robledo LM, Dartigues JF. Frailty among community-dwelling elderly people in France: the three-city study. Journals of Gerontology Series A-Biological Sciences and Medical Sciences. 2008; 63:1089-1096.

Baltes, PB.; Baltes, MM. Successful aging: perspectives from the behavioral sciences. Cambridge University Press; New York: 1990.

Bandeen-Roche K, Xue QL, Ferrucci L, Walston J, Guralnik JM, Chaves P, Zeger SL, Fried LP. Phenotype of frailty: characterization in the women's health and aging studies. Journals of Gerontology Series A-Biological Sciences and Medical Sciences. 2006; 61:262-266.

Banks, J.; Karlsen, S.; Oldfield, Z. Socio-economic position. In: Marmot, M.; Banks, J.; Blundell, R.; Lessof, C.; Nazroo, J., editors. Health, wealth and lifestyles of the older population in England. Institute of Fiscal Studies; London: 2003. p. 71-125.

Barzilay JI, Blaum C, Moore T, Xue QL, Hirsch CH, Walston JD, Fried LP. Insulin resistance and inflammation as precursors of frailty: the Cardiovascular Health Study. Archives of Internal Medicine. 2007; 167:635-641. [PubMed: 17420420]

Baylis D, Bartlett DB, Syddall HE, Ntani G, Gale CR, Cooper C, Lord JM, Sayer AA. Immuneendocrine biomarkers as predictors of frailty and mortality: a 10-year longitudinal study in community-dwelling older people. Age (Dordr. ). 2012 
Bergman H, Ferrucci L, Guralnik J, Hogan DB, Hummel S, Karunananthan S, Wolfson C. Frailty: an emerging research and clinical paradigm--issues and controversies. Journals of Gerontology Series A-Biological Sciences and Medical Sciences. 2007; 62:731-737.

Bouillon K, Sabia S, Jokela M, Gale CR, Singh-Manoux A, Shipley MJ, Kivimaki M, Batty GD. Validating a widely used measure of frailty: are all sub-components necessary? Evidence from the Whitehall II cohort study. Age (Dordr. ). 2012

Boyle PA, Buchman AS, Bennett DA. Purpose in life is associated with a reduced risk of incident disability among community-dwelling older persons. American Journal of Geriatric Psychiatry. 2010; 18:1093-1102. [PubMed: 20808115]

Brayne C, Nickson J, McCracken C, Gill C, Johnson AL. Cognitive function and dementia in six areas of England and Wales: the distribution of MMSE and prevalence of GMS organicity level in the MRC CFA study. Psychological Medicine. 1998; 28:319-335. [PubMed: 9572090]

Chida Y, Steptoe A. Positive psychological well-being and mortality: a quantitative review of prospective observational studies. Psychosomatic Medicine. 2008; 70:741-756. [PubMed: 18725425]

Collins AL, Goldman N, Rodriguez G. Is positive well-being protective of mobility limitations among older adults? Journals of Gerontology Series B-Psychological Sciences and Social Sciences. 2008; 63:321-327.

Espinoza SE, Jung I, Hazuda H. Frailty transitions in the San Antonio Longitudinal Study of Aging. Journal of the American Geriatrics Society. 2012; 60:652-660. [PubMed: 22316162]

Fillit H, Butler RN, Fillit H, Butler RN. The frailty identity crisis. Journal of the American Geriatrics Society. 2009; 57:348-352. [PubMed: 19207150]

Fried LP, Tangen CM, Walston J, Newman AB, Hirsch C, Gottdiener J, Seeman T, Tracy R, Kop WJ, Burke G, McBurnie MA. Frailty in older adults: evidence for a phenotype. Journals of Gerontology Series A-Biological Sciences and Medical Sciences. 2001; 56:M146-M156.

Gale CR, Baylis D, Cooper C, Sayer AA. Inflammatory markers and incident frailty in men and women: the English Longitudinal Study of Ageing. Age (Dordr. ). 2013

Gale, CR.; Deary, IJ.; Stafford, M. A lifecourse approach to psychological and social wellbeing. In: Kuh, D.; Ben-Shlomo, Y.; Hardy, R.; Richards, M.; Cooper, R.; Ferrucci, L., editors. A lifecourse approach to healthy ageing. Oxford University Press; Oxford: (in press)

Gerstorf D, Lovden M, Rocke C, Smith J, Lindenberger U. Well-being affects changes in perceptual speed in advanced old age: Longitudinal evidence for a dynamic link. Developmental Psychology. 2007; 43:705-718. [PubMed: 17484582]

Gill TM, Gahbauer EA, Allore HG, Han L. Transitions between frailty states among community-living older persons. Archives of Internal Medicine. 2006; 166:418-423. [PubMed: 16505261]

Graig, R.; Deverill, C.; Pickering, K. Quality control of blood, saliva and urine analytes. In: Spronston, K.; Mindell, J., editors. Health Survey for England 2004, methodology and documentation. Vol. Volume 2. The Information Centre; London: 2006. p. 34-41.

Hyde M, Wiggins RD, Higgs P, Blane DB. A measure of quality of life in early old age: the theory, development and properties of a needs satisfaction model (CASP-19). Aging \& Mental Health. 2003; 7:186-194. [PubMed: 12775399]

Joint Health Surveys Unit. Health Survey for England physical activity validation study: substantive report. Information Centre for Health and Social Care; Leeds: 2007.

Kiecolt-Glaser JK, McGuire L, Robles TF, Glaser R. Emotions, morbidity, and mortality: new perspectives from psychoneuroimmunology. Annual Review of Psychology. 2002; 53:83-107.

Levy BR, Slade MD, Kasl SV. Longitudinal benefit of positive self-perceptions of aging on functional health. Journals of Gerontology Series B-Psychological Sciences and Social Sciences. 2002; 57:409-417.

Marmot, M.; Nazroo, J.; Banks, J.; Blundell, R.; Erens, B.; Lessof, C.; Huppert, FA. English Longitudinal Study of Ageing: Wave 0 (1998, 1999 and 2001) and Waves 1-4 (2002-2009) [computer file]. 15th Edition. UK Data Archive [distributor]; Colchester, Essex: 2011. SN: 5050

Ostir GV, Berges IM, Ottenbacher ME, Clow A, Ottenbacher KJ. Associations between positive emotion and recovery of functional status following stroke. Psychosomatic Medicine. 2008; 70:404-409. [PubMed: 18434490] 
Ostir GV, Markides KS, Black SA, Goodwin JS. Emotional well-being predicts subsequent functional independence and survival. Journal of the American Geriatrics Society. 2000; 48:473-478. [PubMed: 10811538]

Ostir GV, Ottenbacher KJ, Markides KS. Onset of frailty in older adults and the protective role of positive affect. Psychology and Aging. 2004; 19:402-408. [PubMed: 15382991]

Park-Lee E, Fredman L, Hochberg M, Faulkner K. Positive affect and incidence of frailty in elderly women caregivers and noncaregivers: results of Caregiver-Study of Osteoporotic Fractures. Journal of the American Geriatrics Society. 2009; 57:627-633. [PubMed: 19392954]

Puts MT, Visser M, Twisk JW, Deeg DJ, Lips P. Endocrine and inflammatory markers as predictors of frailty. Clinical Endocrinology (Oxford). 2005; 63:403-411. [PubMed: 16181232]

Reiner AP, Aragaki AK, Gray SL, Wactawski-Wende J, Cauley JA, Cochrane BB, Kooperberg CL, Woods NF, Lacroix AZ. Inflammation and thrombosis biomarkers and incident frailty in postmenopausal women. American Journal of Medicine. 2009; 122:947-954. [PubMed: 19682668]

Rockwood K, Bergman H. FRAILTY: A Report from the 3(rd) Joint Workshop of IAGG/WHO/ SFGG, Athens, January 2012. Canadian Geriatrics Journal. 2012; 15:31-36. [PubMed: 23259017]

Rockwood K, Fox RA, Stolee P, Robertson D, Beattie BL. Frailty in elderly people: an evolving concept. Canadian Medical Association Journal. 1994; 150:489-495. [PubMed: 8313261]

Rodriguez-Manas L, Feart C, Mann G, Vina J, Chatterji S, Chodzko-Zajko W, Gonzalez-Colaco HM, Bergman H, Carcaillon L, Nicholson C, Scuteri A, Sinclair A, Pelaez M, Van der Cammen T, Beland F, Bickenbach J, Delamarche P, Ferrucci L, Fried LP, Gutierrez-Robledo LM, Rockwood K, Rodriguez AF, Serviddio G, Vega E. Searching for an operational definition of frailty: a Delphi method based consensus statement: the frailty operative definition-consensus conference project. Journals of Gerontology Series A-Biological Sciences and Medical Sciences. 2013; 68:62-67.

Roth, M.; Huppert, FA.; Mountjoy, CQ.; Tym, E. The revised Cambridge examination for mental disorders of the elderly. Cambridge University Press; Cambridge: 1999.

Rowe, JW.; Kahn, RL. Successful aging: The MacArthur Foundation Study. Pantheon Books; New York: 1998.

Royston P. Multiple imputation of missing values. Stata Journal. 2004; 4:227-241.

Steel, N.; Huppert, FA.; McWilliams, BMD. Physical and cognitive function. In: Marmot, M.; Banks, J.; Blundell, R.; Lessof, C.; Nazroo, J., editors. Health, wealth and lifestyles of the older population in England: the 2002 English Longitudinal Study of Ageing. Institute of Fiscal Studies; London: 2003. p. 249-300.

Steffick, DE.; The HRS working group. Documentation of affective functioning measures in the Health and Retirement Study. HRS/AHEAD Documentation Report DR-005. 2000. [online report]

Steptoe A, Breeze E, Banks J, Nazroo J. Cohort Profile: The English Longitudinal Study of Ageing. International Journal Epidemiology. 2012a

Steptoe A, Demakakos P, de OC, Wardle J. Distinctive biological correlates of positive psychological well-being in older men and women. Psychosomatic Medicine. 2012b; 74:501-508. [PubMed: 22511728]

Steptoe A, Dockray S, Wardle J. Positive Affect and Psychobiological Processes Relevant to Health. Journal of Personality. 2009; 77:1747-1776. [PubMed: 19796062]

Syddall H, Roberts HC, Evandrou M, Cooper C, Bergman H, Aihie SA. Prevalence and correlates of frailty among community-dwelling older men and women: findings from the Hertfordshire Cohort Study. Age Ageing. 2010; 39:197-203. [PubMed: 20007127]

Taylor, R.; Conway, L.; Calderwood, L.; Lessof, C. Methodology. In: Marmot, M.; Banks, J.; Blundell, R.; Lessof, C.; Nazroo, J., editors. Health, wealth and lifestyles of the older population in England: the 2002 English Longitudinal Study of Ageing. Institute of Fiscal Studies; London: 2003. p. 357-374.

Walston J, Hadley EC, Ferrucci L, Guralnik JM, Newman AB, Studenski SA, Ershler WB, Harris T, Fried LP. Research agenda for frailty in older adults: toward a better understanding of physiology and etiology: summary from the American Geriatrics Society/National Institute on Aging Research Conference on Frailty in Older Adults. Journal of the American Geriatrics Society. 2006; 54:991-1001. [PubMed: 16776798] 

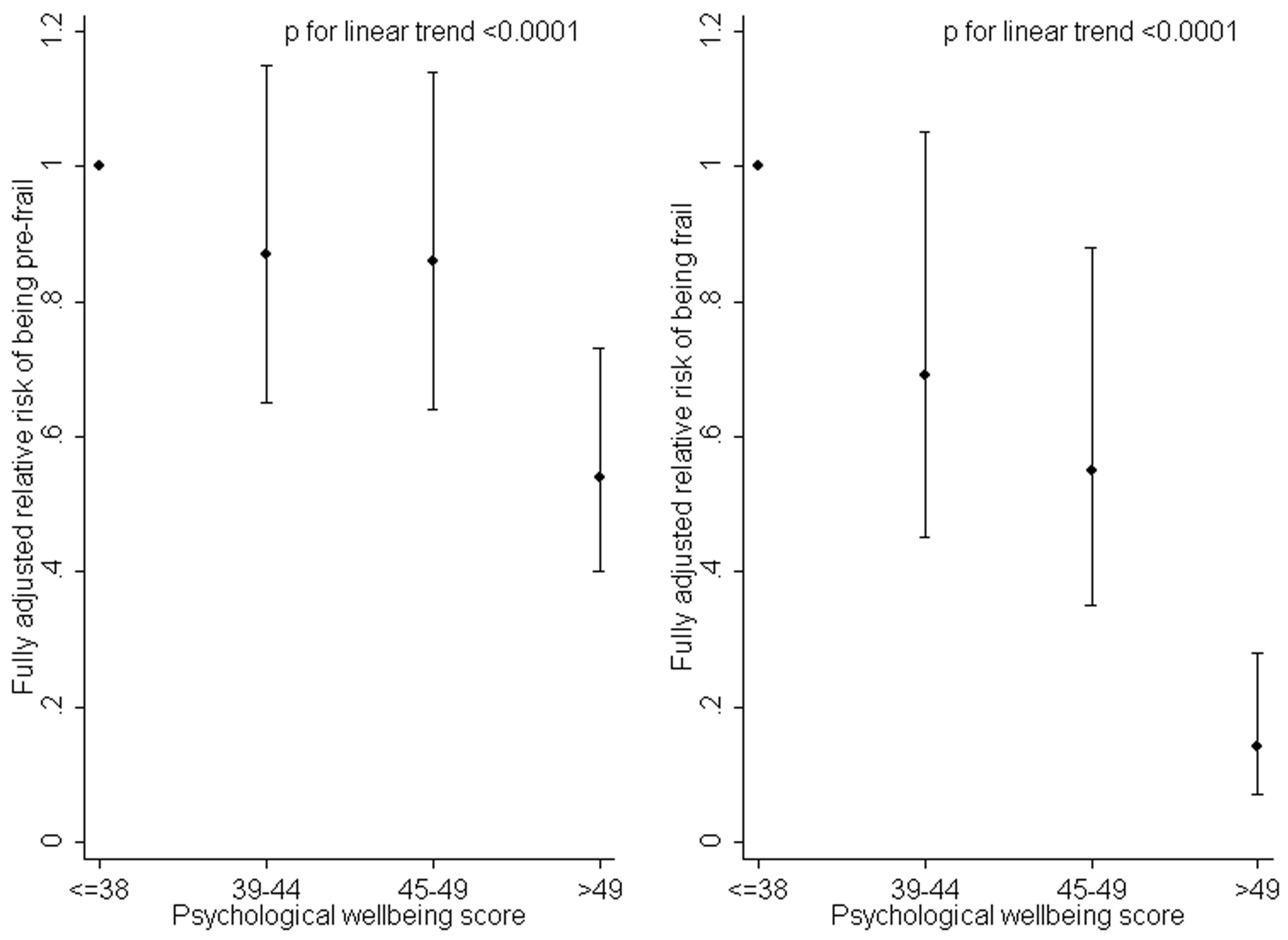

Figure 1.

Fully adjusted relative risk ratios for being pre-frail or frail at follow-up according to quarters of the distribution of psychological wellbeing at baseline. People in the lowest quarter of the distribution are the reference group 


\section{Table 1}

Baseline characteristics of the study participants $(n=2557)$ according to frailty status at follow-up

\begin{tabular}{|c|c|c|c|c|c|}
\hline & $\begin{array}{l}\text { Not frail } \\
(n=1186)\end{array}$ & $\begin{array}{l}\text { Pre-frail } \\
(n=1058)\end{array}$ & $\begin{array}{l}\text { Frail } \\
(n=313)\end{array}$ & $\begin{array}{l}\text { P for difference } \\
\text { between not frail and } \\
\text { pre-frail }\end{array}$ & $\begin{array}{l}P \text { for difference } \\
\text { between pre-frail } \\
\text { and frail }\end{array}$ \\
\hline Female, $(\%)$ & 52.1 & 55.5 & 65.2 & 0.13 & 0.006 \\
\hline Lowest quintile of wealth, $(\%)$ & 9.24 & 19.1 & 35.3 & $<0.0001$ & $<0.0001$ \\
\hline No of chronic diseases, mean (SD) & $0.98(1.09)$ & $1.43(1.18)$ & $2.08(1.15)$ & $<0.0001$ & $<0.0001$ \\
\hline Depressive symptom score $\geq 4, \%$ & 4.57 & 11.5 & 21.2 & $<0.0001$ & $<0.0001$ \\
\hline Cognitive function, mean (SD) & $30.5(5.57)$ & $27.9(5.50)$ & $25.1(5.21)$ & $<0.0001$ & $<0.0001$ \\
\hline Eudaimonic wellbeing score, mean (SD) & $35.3(6.47)$ & $32.7(6.79)$ & $28.6(6.22)$ & $<0.0001$ & $<0.0001$ \\
\hline No of frailty criteria present, mean (SD) & $0.29(0.61)$ & $0.81(0.90)$ & $1.79(1.14)$ & $<0.0001$ & $<0.0001$ \\
\hline
\end{tabular}




\section{Table 2}

Relative risk ratios $(95 \% \mathrm{CI})$ for incident pre-frailty or frailty for a standard deviation increase in psychological wellbeing scores at baseline.

\begin{tabular}{|c|c|c|c|c|}
\hline \multirow[t]{2}{*}{$\begin{array}{l}\text { Psychological wellbeing } \\
\text { scores, per SD increase }\end{array}$} & \multicolumn{2}{|c|}{ RR $(95 \% \mathrm{CI})$, adjusted for age, sex, \& baseline frailty status } & \multicolumn{2}{|c|}{ RR $(95 \%$ CI $)$, fully adjusted ${ }^{2}$} \\
\hline & Pre-frailty & Frailty & Pre-frailty & Frailty \\
\hline Total score & $0.69(0.63,0.77)$ & $0.46(0.40,0.54)$ & $0.79(0.71,0.89)$ & $0.62(0.52,0.74)$ \\
\hline Hedonic wellbeing & $0.75(0.68,0.83)$ & $0.58(0.50,0.67)$ & $0.83(0.74,0.92)$ & $0.70(0.59,0.82)$ \\
\hline Eudaimonic wellbeing & $0.70(0.63,0.78)$ & $0.47(0.40,0.55)$ & $0.76(0.72,0.90)$ & $0.64(0.53,0.76)$ \\
\hline
\end{tabular}

1 adjusted for age, sex, household wealth, depressive symptoms, cognitive function, body mass index, smoking, number of chronic physical diseases and whether frail, pre-frail or non-frail at baseline. 